\section{The Effect of Gold Nanoparticles on Electrical Impedance of Tissue on Low Frequency Ranges}

\author{
Ostovari M. ${ }^{1,2}$, Riahi Alam N.3, Zabihzadeh M. ${ }^{1,2,4 *}$, Gharib- \\ vand M. M. ${ }^{5}$, Hoseini-Ghahfarokhi M. ${ }^{1}$
}

\begin{abstract}
Introduction: Electrical impedance of tissues on low frequencies includes useful information about functional and structural changes in tissues. This property is used in Electrical Impedance Tomography (EIT) imaging modality for the detection of lesions in tissues.
\end{abstract}

Objective: The goal of this article is to study changes in electrical impedance of tissues in the presence of gold nanoparticles.

Materials and Methods: Spherical gold nanoparticles with size of 20$25 \mathrm{~nm}$ were synthesized with Turkevich method. Size distribution and shape of nanoparticles were characterized by transmission electron microscopy (TEM). Electrical impedance of two types of phantoms (chicken fat and muscle paste tissues) was measured by 4-electrode method with and without gold nanoparticles.

Results: Results demonstrate a reduction in electrical impedance of tissues in the presence of gold nanoparticles. However, this reduction is not the same for fat and

muscle tissues. Reductions in resistive impedance are $40.24 \Omega$ and $1.93 \Omega$ for fat and muscle tissues on the frequency of $1 \mathrm{KHz}$, respectively. A reduction in electrical impedance is accompanied by a rise in electrical conductance leading to increase in EIT signal.

Conclusion: As signal enhancement is different for fat and muscle tissues; presence of gold nanoparticles could be used to improve EIT image contrast.

\section{Keywords}

Contrast Agent, Electrical Impedance, Gold Nanoparticle, Medical Imaging

\section{Introduction}

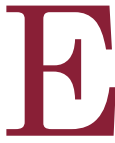

lectrical properties of biological tissues include valuable functional information of tissues and have their pathological information at cellular levels $[1,2]$. Electrical impedance measurement is a low-cost, fast and minimally invasive method to determine these properties of tissues. One of the imaging techniques based on this method is known as electrical impedance tomography (EIT) [3, 4]. EIT maps conductance distribution of tissue inside the body. This imaging technique helps to detect changes in the impedance of tissue lesions even when their structures show no changes, and thereby, the functional image of tissue is achievable $[5,6]$. This technique has advantages of ignoring the use of ionizing radiation and its temporal resolution is interested in order of milliseconds.

A small amount of signal-to-noise ratio is an important problem in bio-
${ }^{1}$ Nanotechnology Re-

search Center, Ahvaz

Jundishapur University

of Medical Sciences,

Ahvaz, Iran

${ }^{2}$ Department of Medical

Physics, School of Medi-

cine, Ahvaz Jundishapur

University of Medical

Sciences, Ahvaz, Iran

${ }^{3}$ Department of Medical

Physics and Biomedi-

cal EngineeringTehran

University of Medical

Sciences Tehran Iran

${ }^{4}$ Department of Clinical

Oncology, Golestan Hos-

pital, Ahvaz Jundishapur

University of Medical

Sciences, Ahvaz, Iran

${ }^{5}$ Department of Radiol-

ogy, Golestan Medical

Center, Ahvaz Jundis-

hapur University of Medi-

cal Sciences, Ahvaz, Iran

*Corresponding author:

M. Zabihzadeh

Nanotechnology Re-

search Center, Ahvaz

Jundishapur University

of Medical Sciences,

Ahvaz, Iran

E-mail: manzabih@

gmail.com

Received: 14 August 2017

Accepted: 12 October 2017 
logical image processing. Many imaging modalities such as magnetic resonance imaging (MRI) and computed tomography (CT) use some agents (sensitizers) with special properties to increase SNR. For example, this special property is an atomic number in CT (typically iodine or barium compounds with high atomic number) [7] or is magnetization level in MRI (typically components with Gadolinium as paramagnetic metal ion)[8]. Recently, verity components of Gold nano-particles (Au NP) have been of interest to be used in CT as a contrast agent $[9,10]$ or in radiotherapy as a dose enhancer agent [11-13]. Theoretically, such an agent in EIT should affect electrical impedance of tissues. Some researchers demonstrated that presence of Au NP in Deionized water (DI) solution reduces electrical impedance of DI water [14-16]. However, in contrast to biological tissues, DI water does not have capacitive impedance and is almost free of charge carriers. Having capacitive impedance and the presence of charge carriers in biological tissues might have a noticeable effect on electrical behavior of Au NPs. Therefore, studying electrical behavior of Au NPs in a tissue-like environment is an important step to evaluate the potential use of Au NPs as a contrast agent for EIT technique.

Different phantoms have been used so as to study electrical properties of tissues [17-19]. Based on research literature, a chicken tissue phantom is a recommended phantom in this regard [19-21]. Therefore, the effect of $\mathrm{Au}$ NPs on electrical impedance of chicken tissue phantom is explored in this study. EIT signal on frequencies between $10 \mathrm{~Hz}$ and $10 \mathrm{kHz}$ is more sensitive to cellular structure tissues than frequencies above $10 \mathrm{kHz}$ [22]. From literature, the 4-electrode method is a standard and comfortable method for the impedance measurement [23].

To investigate the possibility of using $\mathrm{Au}$ NPs as a contrast agent in EIT, well-known of electrical properties of tissues in the presence of Au NPs are necessitated. In this study,
EI measurement of synthesized Au NPs in a homemade chicken tissue phantom was done on current frequencies between $10 \mathrm{~Hz}$ to 10 $\mathrm{kHz}$ with 4-electrode method.

\section{Materials and Methods}

\section{Electrical Impedance}

Biological tissues can be considered as a combination of three distinct mediums; intracellular medium (ICM), Extracellular medium (ECM) and cell membrane (CM). ICM and ECM consist of ions and other charged particles and they are electrically conductive [24]. $\mathrm{CM}$ is made up of a lipid layer sandwiched between two protein layers and its electrical response is as a capacitor. Capacitive impedance (X) of CM has inverse relation with the frequency of applied voltage $X=1 / \omega C$. On low

frequency, its impedance becomes large and notable. So, a tiny current can flow through it and EI of tissue on this frequency region depending on electrical impedance of ECM and structural configuration of CMs. On high frequency, capacitive impedance of CM becomes very low and CM behaves as a short circuit. Accordingly, EI of tissue depends on electrical impedance of ICM and ECM and less dependent on the structural configuration of CMs. Electrical impedance is a complex quantity, $Z(f)=Z_{R}(f)-i Z_{C}(f)=|Z| e^{i \theta}$ in which its real part shows resistive impedance $\left(Z_{R}\right)$ or Resistance $(R)$ and its imaginary part represents capacitive impedance $\left(Z_{C}\right)$ or Reactance $(X)$. Amplitude of impedance is calculated from division of voltage to current $|Z(f)|=V(f) / I(f)$ and angle $\theta$ shows the phase difference between voltage and current.

$$
\begin{aligned}
& Z_{R}=R=|Z| \cos \theta ;[\Omega] \\
& Z_{C}=X=|Z| \operatorname{Sin} \theta ;[-\Omega]
\end{aligned}
$$


Different methods are used to measure electrical impedance of biological tissues. 4-electrode method is a standard and reliable method which is used in some research studies as a gold standard for the measurement [23]. This method as shown in Figure 1, was used to determine the electrical impedance.

From Figure 1, the phantom with volume of $A B H\left[\mathrm{~m}^{3}\right]$ connected to an alternating current supply and is series with of a determined Resistor $(R)$. The electric current of passing through the circuit was calculated as $I=V_{2} / R$ where $V_{2}$ is voltage that is measured at both ends of resistor $(R)$. Sinusoidal electric current was injected (within a frequency range of $10 \mathrm{~Hz}$ to $10 \mathrm{kHz}$ ) to phantom by 2 stainless steel electrodes at both ends of it and voltage $V_{1}$ was measured by 2 needle-like electrodes placed in the middle of phantom and separated at a distance of $L[\mathrm{~m}]$. Specific resistive impedance and conductance of tissue were calculated through equation 3 :

$$
R=\rho L / S \Rightarrow \rho=\frac{R \times S}{L}[\Omega . m]
$$

Where $\rho$ is specific resistance, $L$ is the distance between electrodes that measure voltage, and $S$ is the cross-section area of sample tissue $S=B \times H \quad\left[\mathrm{~m}^{2}\right]$.

$$
\sigma=\frac{1}{\rho}=\frac{L}{R \times S} \quad[S / m]
$$

Where $\sigma$ is specific conductance of tissue.

The parameters are considered as $L=10 \mathrm{~mm}$ , $A=50 \mathrm{~mm}, B=20 \mathrm{~mm}, H=20 \mathrm{~mm}$ and $R=980 \Omega$. The Voltage and frequency were measured with Goodwill GDS-1072B oscilloscope, resistor R was measured with KAISE digital multi-meter sk-6111 and current of circuit was checked with PHYWE ampere meter.

Gold Nano-particle (Au NP) Synthesis $\mathrm{Au}$ NPs were synthesized with Turkevich

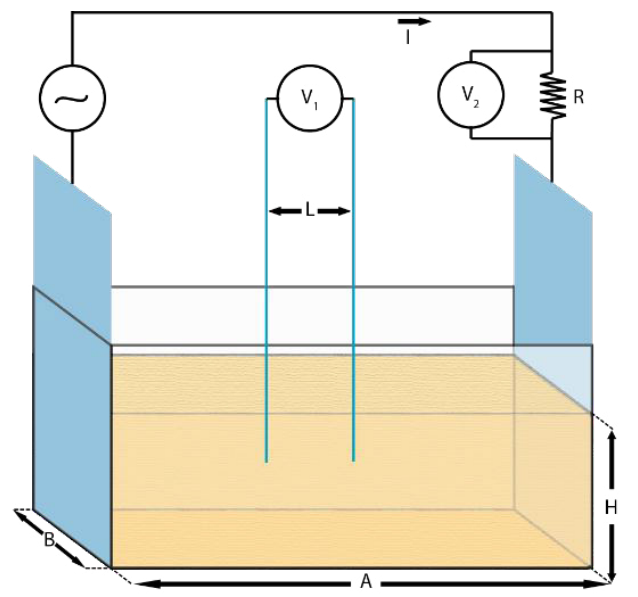

Figure 1: Diagram of a measurement device based on the 4-electrode method [23].

method [25], in which reduction of chloroauric acid (HAuCl4) was done by sodium citrate. Chloroauric acid and sodium citrate were obtained from Sigma-Aldrich, USA. As a brief description, chloroauric acid was heated and allowed to boil with strong stirring (300 rpm) in a $250 \mathrm{ml}$ two-necked round bottom flask immersed in an oil bath and equipped with a condenser. While the solution was boiling, 2 $\mathrm{ml}$ of $34 \mathrm{mM}$ sodium citrate was rapidly added to it which to cause the color of solution varying from black to wine red and Au NPs with controlled size start to synthesize. Afterwards, colloidal solution was cold slowly to reach room temperature. Size distribution and shape of synthesized Au NPs were characterized by transmission electron microscopy (TEM) (Zeiss-EM10C-100 KV). Moreover, UV-Visible absorption spectrum of Au NPs was obtained by Spekol 1300 spectrophotometer (Analytik Jena, Germany).

\section{Phantoms Preparation and Measure- ments}

There are different types of phantoms that are used in various researches for studying electrical behavior of tissues and electrical properties of Au NPs. Electrical properties of Au NPs were explored via DI water phantom in most investigations. As real tissues have capaci- 
tive impedance, a phantom made of substance which has capacitive impedance is more suitable than only resistive phantoms (DI water). Some researches show that a chicken tissue phantom which has both capacitive and resistive impedance, is a good phantom for the simulation of electrical properties of human tissues $[19,20]$. Two types of phantoms are used in this article, the chicken muscle paste phantom with volume $(50 \mathrm{~mm} \times 20 \mathrm{~mm} \times 20 \mathrm{~mm})$ and the chicken fat block phantom with volume $(5 \mathrm{~mm} \times 20 \mathrm{~mm} \times 15 \mathrm{~mm})$. Chicken tissues were bought from markets; all bones were removed carefully, and cut in small pieces. Muscle and fat tissues were washed separately with deionized water (MiliQ water). After three times washing, the external tissue water was removed by keeping them in a kitchen strainer. $2 \%$ deionized water was added to the chicken muscle pieces and minced for 2 minutes in a mixture grinder (2400 rpm). Again 1\% DI water was added to the mixture and grind for 2 minutes, then, it was kept in refrigerator for 10 hours. In total, 6 identical phantoms for each material were prepared, and the experiment was repeated with 3 phantoms including $\mathrm{Au}$

NPs (with concentration of $0.15 \frac{\mathrm{mg}}{\mathrm{ml}}$ ) and 3 phantoms without Au NPs. Reported data for voltage and current in each case is average of these measurements. Before the measurement, for the phantom-loaded $\mathrm{Au} \mathrm{NPs}$, the determined volume of Au NPs ( $650 \mu \mathrm{l}$ and $50 \mu \mathrm{l}$ for muscle and fat phantoms, respectively) was divided to 10 parts and each part injected to different depths of phantom and then allowed to diffuse within the tissues for $2 \mathrm{~min}$ utes. After that, the measurement was done.

\section{Results and Discussion}

TEM image confirmed that size of synthesized Au NPs is about 20-25 nm; they have a spherical shape (Figure 2. a). The UV-Visible spectrum also approved the size distribution by $525 \mathrm{~nm}$ peak in the absorption pattern (Figure 2. b).

Amplitude of electrical impedance and phase angle between voltage and current flowed through phantoms were measured on different frequencies. Also, Resistive impedance (RI), Capacitive impedance (CI), Specific resistance $(\rho)$ and specific conductance $(\sigma)$ of phantoms were calculated based on equations $1,2,3$ and 4, and variations of all these parameters in terms of frequency were plotted in Figures 3, 4 and 5.

Results reveal that resistive impedance a)

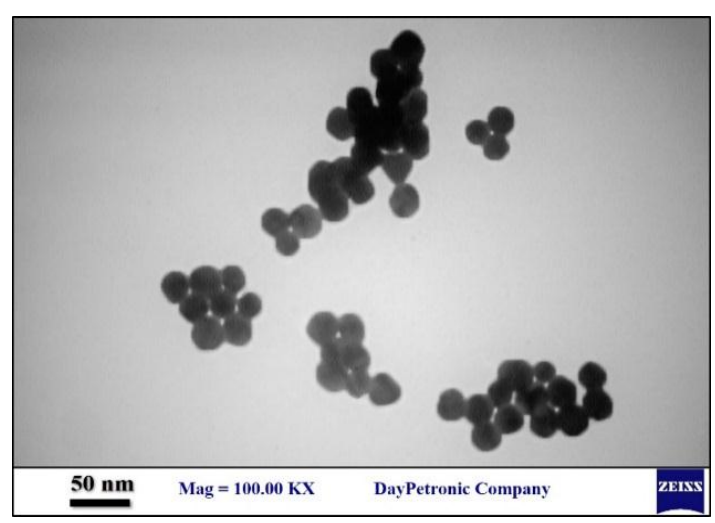

b)

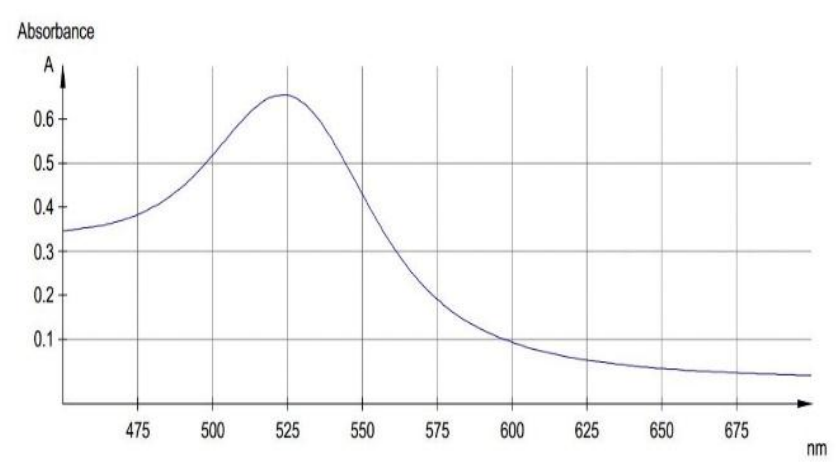

Figure 2: a. TEM image and b. UV-visible spectrum of synthesized Au NPs. 
of fat is more than resistive impedance of muscle (Figures 3.c and 4.c and 5.a), and the

a)

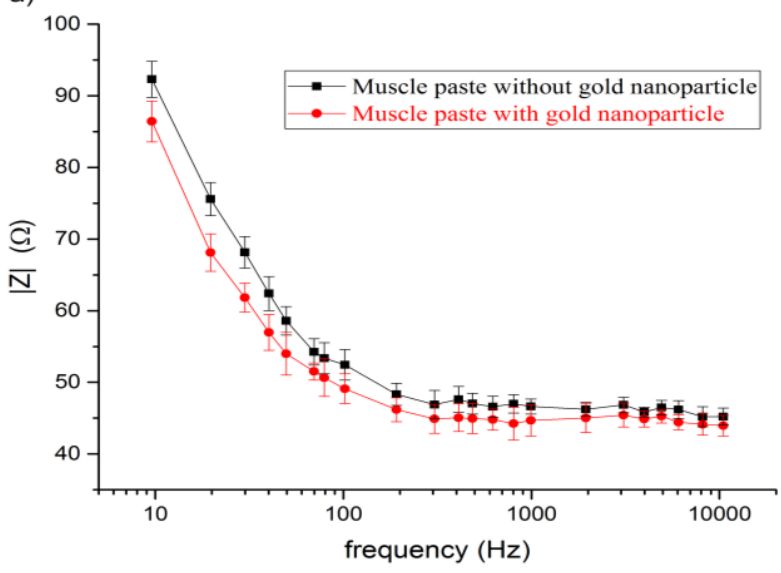

c)

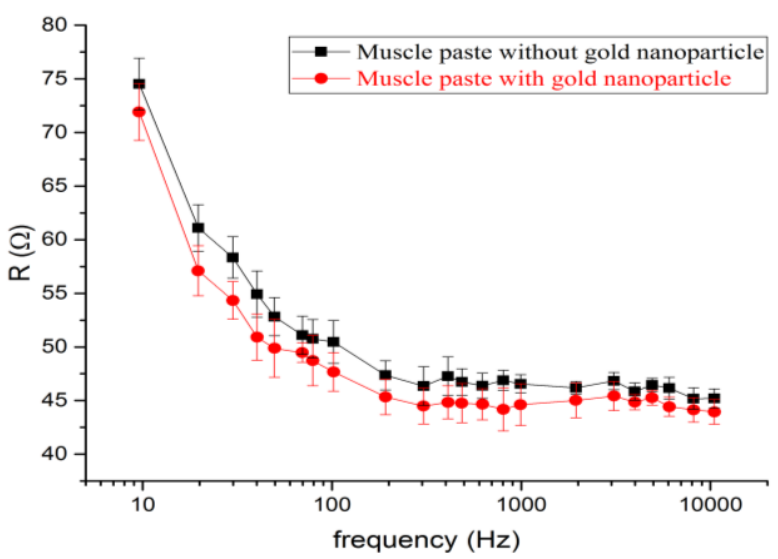

e)

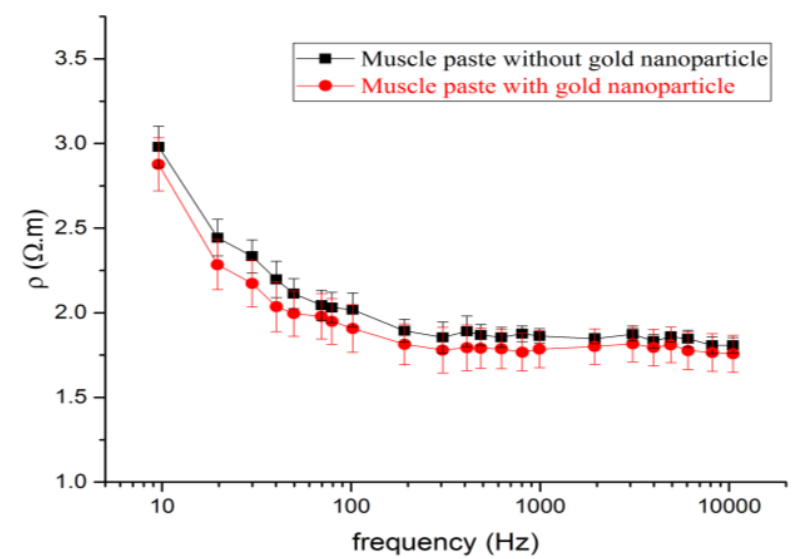

presence of $\mathrm{Au}$ NPs leads to decrease in resistive impedance of both of them (reduction

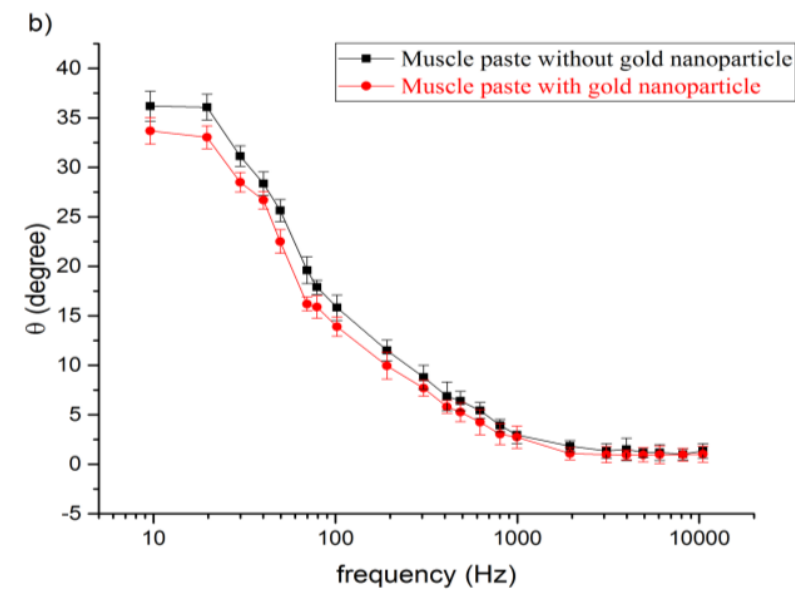

d)
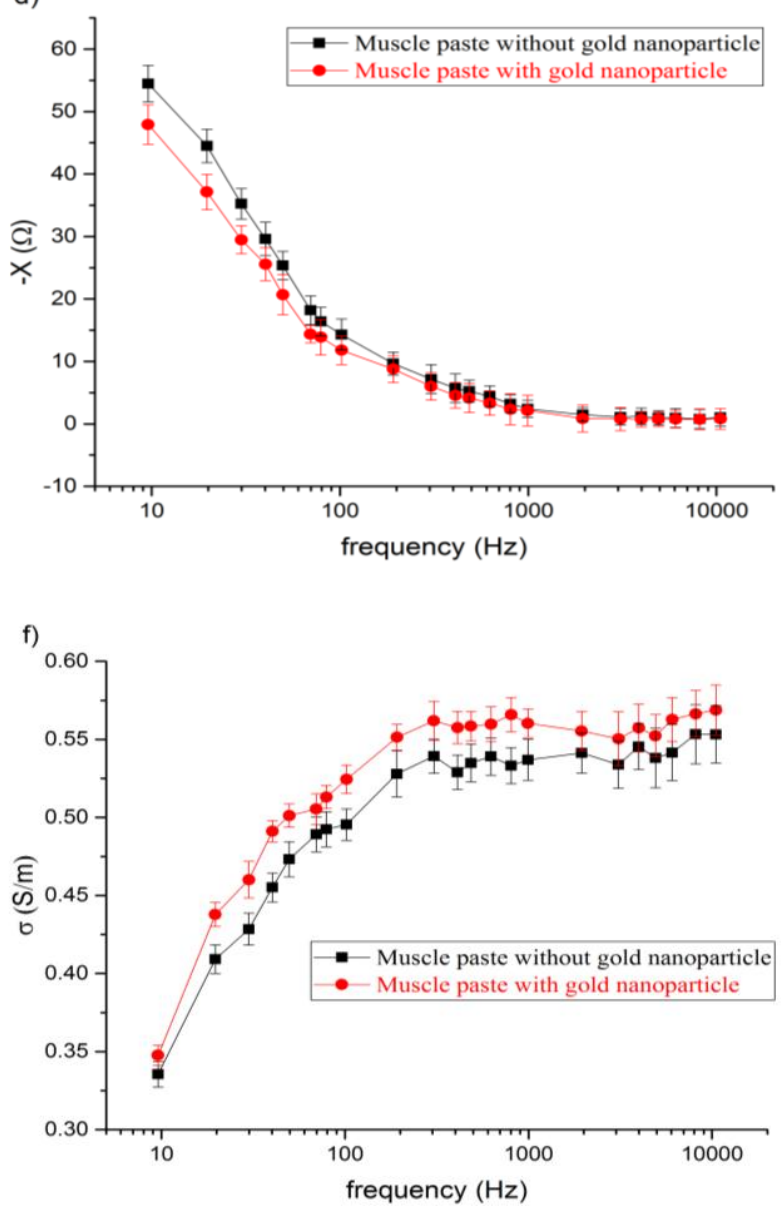

Figure 3: Electrical properties of muscle paste phantom with and without Au NPs; the frequency dependence of $a$. the impedance amplitude, b. the phase difference, c.the resistive impedance, $d$. the capacitive impedance, e. the specific impedance, and $f$. the specific conductance. 

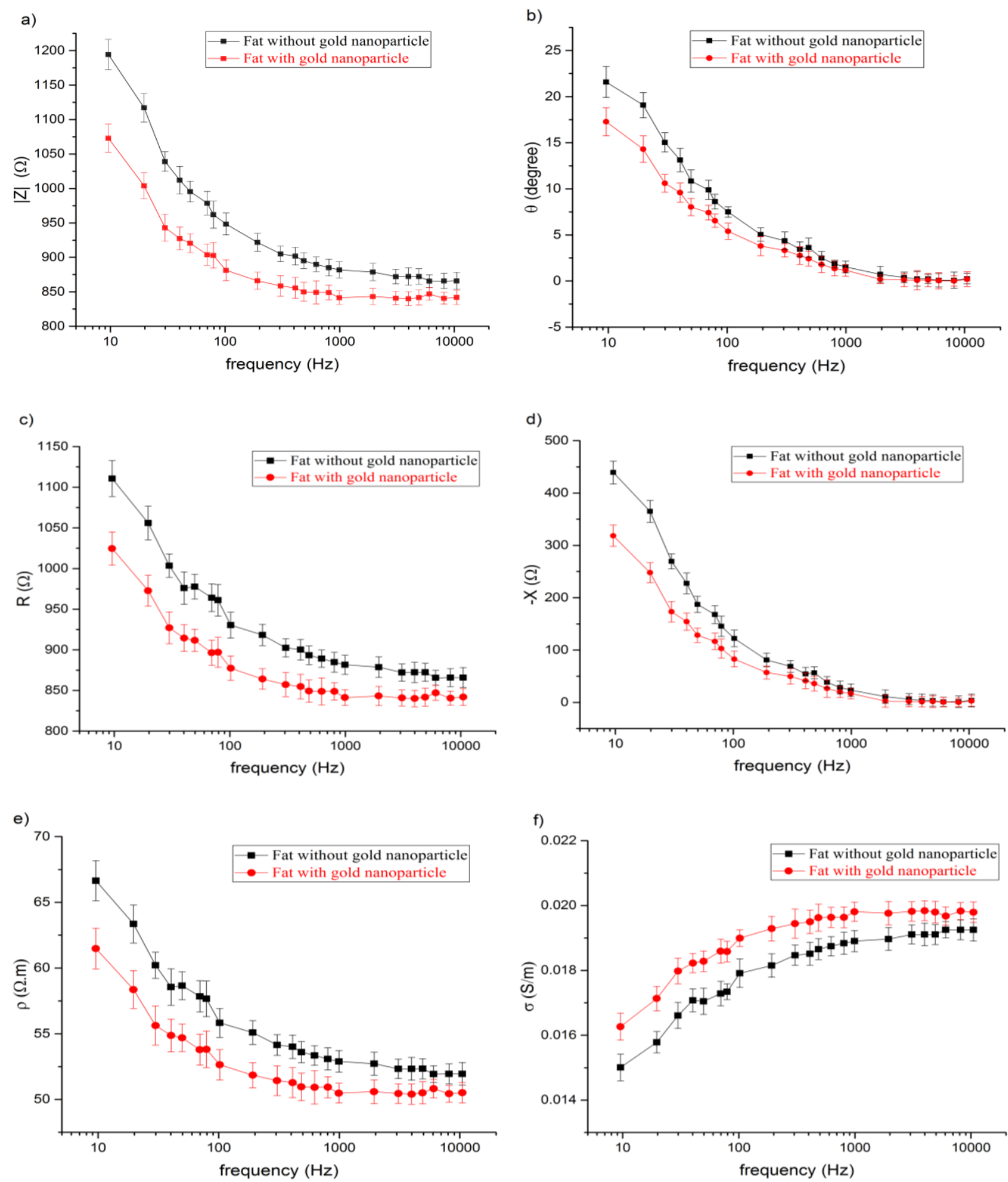

Figure 4: Electrical properties of fat phantom with and without Au NPs; frequency dependence of a. impedance amplitude, b. phase difference, c. resistive impedance, d. capacitive impedance, e. specific impedance, and f. specific conductance.

of resistive impedance of tissues on the frequency $1 \mathrm{KHz}$ because gold nanoparticles are $1.93 \Omega$ and $40.24 \Omega$ for muscle and fat tissues, respectively. However, this reduction in fat tissue is more than muscle tissue. Dissimilarity between resistive impedance of fat and muscle 
tissue can be attributed to the difference in the ionic content and ionic mobility of these tissues. More ionic content and the easy movement of ions in muscle tissues compared to fat tissue may lead to increase in the conduction current and reduction in the resistive impedance of muscle tissue.

In addition to the ionic content and ionic mobility, water content of fat tissue is less than that of muscle. It can cause a decrease in electrical conduction and increase in electrical impedance of fat tissue. However this effect is more significant on high frequency.

A conduction mechanism in Au NPs can be explained by Quantum confinement effects and large surface-to-volume ratio of them [26]. This mechanism can be affected by the presence of ions and other charge carriers in a medium [27]. As an amount of ions and other charge carriers increase, electrical conduction can be dominated by ionic response. Different impedance responses of fat and muscle tissue to Au NPs can also be ascribed to difference in amounts of ions in these tissues.

Capacitive impedance of fat and muscle phantoms without Au NPs do not show remarkable differences on frequencies above

a)

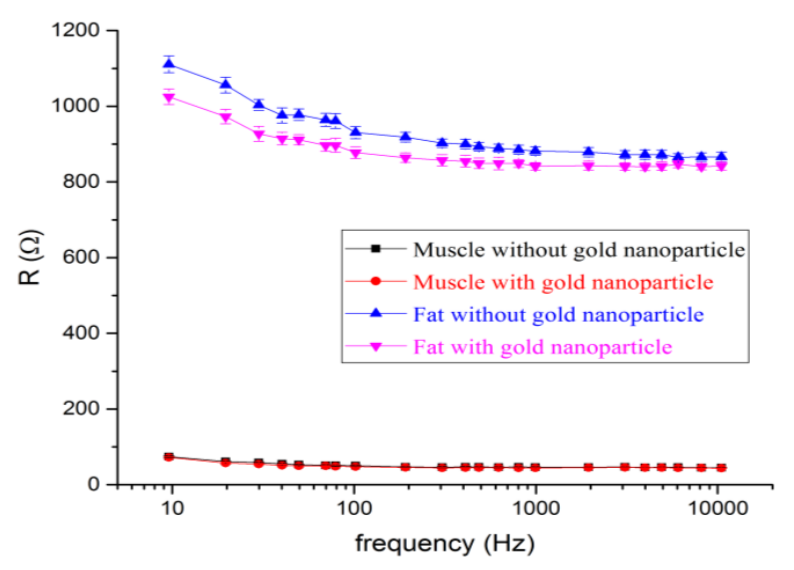

$1 \mathrm{kHz}$, although capacitive impedance of fat phantoms are more than that of muscle phantoms (Figure 5.b). Presence of Au NPs leads to decrease in capacitive impedance of both phantoms (reduction in capacitive impedance of fat and muscle on the frequency $1 \mathrm{KHz}$ are $6.48 \Omega$ and $0.27 \Omega$, respectively). This reduction for fat phantoms is more than that of muscle phantoms (Figures 3.d, 4.d and 5.b).

Capacitive impedance is proportional to the amount of displacement current on a certain frequency in a particular tissue. Displacement current is related to membrane structure of cells and amount of polar molecules such as proteins in the tissue [28].

Cell membrane has protein-lipid-protein (PLP) structure that acts like a capacitor [20]. Difference in structure of cell membranes leads to different amounts of membrane capacitances. Because capacitive impedance has an inverse relation to membrane capacitance

$(X=1 / \omega C)$, increase in membrane capacitance leads to the reduction in capacitive impedance [28].

Polar molecules play a role of electrical dipole moments in an AC electric field. More

b)

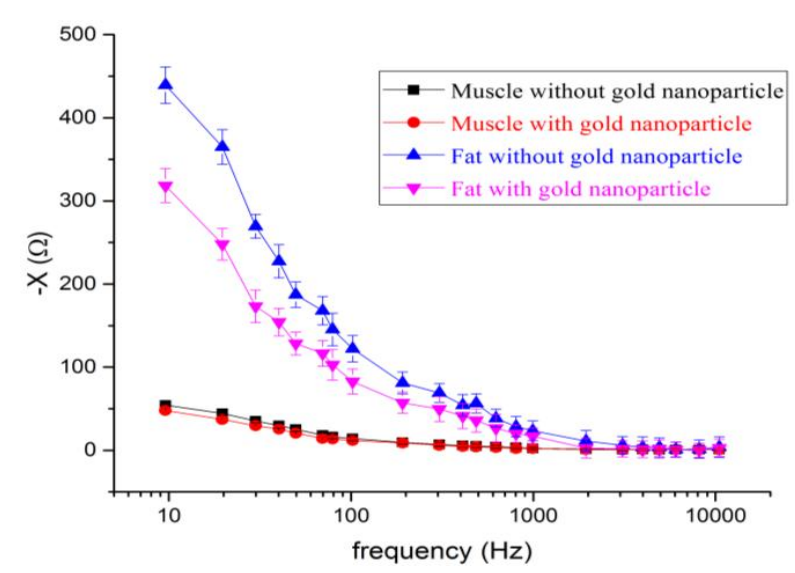

Figure 5: Electrical properties of Fat and muscle paste phantoms with and without Au NPs; the frequency dependence of a. resistive impedance and b. capacitive impedance. 
polar molecules and easily tumbling of these molecules in muscle tissue cause an increase in displacement current and the reduction in capacitive impedance of it compared to fat tissue.

$\mathrm{Au}$ NPs injected in a tissue are covered by ions and other charged particles [27] . Au NP and charged particles concentrated around of it can be considered as an electrical dipole moment. Therefore, Au NPs can affect displacement current and capacitive impedance like polar molecules. As the frequency increases, reorientation of these dipoles with alternative current $(\mathrm{AC})$ electric field becomes more difficult, and their influence on displacement current enhancement becomes restricted. Accordingly, capacitive impedance with and without $\mathrm{Au}$ NPs do not have any remarkable difference on high frequency The reduction in electrical impedance is equivalent to an increase in electrical conductance. As can be seen in prior Figures (Figures 3.f and 4.f), the presence of $\mathrm{Au}$ NPs leads to increase in specific conductance of both fat and muscle phantoms. Considering the difference between conductance of fat and muscle tissues, the presence of Au NPs leads to $4.3 \%$ and $4.7 \%$ increase in conductance on the frequency of $1 \mathrm{kHz}$ for muscle and fat tissues, respectively.

\section{Conclusion}

Difference of electrical impedance between phantoms with and without Au NP on low frequency (between $10 \mathrm{~Hz}-1 \mathrm{kHz}$ ) is more than that of higher frequency (between $1 \mathrm{kHz}-10$ $\mathrm{kHz}$ ). These differences observed in fat phantoms are more than those in muscle paste phantoms. Capacitive and resistive impedances of fat tissue are more than ones from muscle tissue; a decrease in capacitive and resistive impedance of fat tissue is more than those of muscle tissue. As electrical impedance was reduced by presence of Au NPs, the electrical conductance of tissues was increased. Hence, an injection of $\mathrm{Au}$ NPs has the potential to increase the signal intensity of EIT and its sensi- tivity. Different values of signal enhancement for fat and muscle tissues, due to the presence of Au NPs, could be used to improve EIT image contrast.

\section{Acknowledgment}

This study was financially supported by Research Affairs of Ahvaz Jundishapur University of Medical Sciences, Ahvaz, Iran (Grant No. U-95376).

\section{Conflict of Interest}

Authors have no conflicts of interest to declare.

\section{References}

1. Faes TJ, van der Meij HA, de Munck JC, Heethaar RM. The electric resistivity of human tissues $(100 \mathrm{~Hz}-10 \mathrm{MHz}$ ): a meta-analysis of review studies. Physiol Meas. 1999;20:R1-10. PubMed PMID: 10593226.

2. Miklavčič D, Pavšelj N, Hart FX. Electric properties of tissues. Wiley encyclopedia of biomedical engineering. 2006.

3. Borcea L. Electrical impedance tomography. Inverse problems. 2002;18:R99.

4. Liu R, Liu J, Wang G, Ding H, editors. Minimally invasive electrical impedance tomography-promising way to decrease diagnostics uncertainty. Nano/Micro Engineered and Molecular Systems, 2006 NEMS'06 1st IEEE International Conference on; 2006: IEEE.

5. Gong B, Krueger-Ziolek S, Moeller K, Schullcke $B$, Zhao Z. Electrical impedance tomography: functional lung imaging on its way to clinical practice? Expert Rev Respir Med. 2015;9:72137. doi: 10.1586/17476348.2015.1103650. PubMed PMID: 26488464.

6. Lundin S, Stenqvist 0 . Electrical impedance tomography: potentials and pitfalls. Curr Opin Crit Care. 2012;18:35-41. doi: 10.1097/ MCC.0b013e32834eb462. PubMed PMID: 22201705.

7. Lusic H, Grinstaff MW. X-ray-computed tomography contrast agents. Chem Rev. 2013;113:1641-66. doi: 10.1021/cr200358s. PubMed PMID: 23210836; PubMed Central PMCID: PMC3878741.

8. Riyahi-Alam N, Behrouzkia Z, Seifalian A, 
Haghgoo Jahromi S. Properties evaluation of a new MRI contrast agent based on Gdloaded nanoparticles. Biol Trace Elem Res. 2010;137:324-34. doi: 10.1007/s12011-0098587-3. PubMed PMID: 20049554.

9. Cole LE, Ross RD, Tilley JM, Vargo-Gogola T, Roeder RK. Gold nanoparticles as contrast agents in $\mathrm{x}$-ray imaging and computed tomography. Nanomedicine (Lond). 2015;10:32141. doi: 10.2217/nnm.14.171. PubMed PMID: 25600973.

10.Xi D, Dong S, Meng X, Lu Q, Meng L, Ye J. Gold nanoparticles as computerized tomography (CT) contrast agents. Rsc Advances. 2012;2:12515-24.

11.Zabihzadeh M, Arefian S. Tumor dose enhancement by nanoparticles during high dose rate (192)Ir brachytherapy. J Cancer Res Ther. 2015;11:752-9. doi: 10.4103/09731482.153668. PubMed PMID: 26881513.

12.Zabihzadeh $M$, Moshirian T, Ghorbani M, Knaup C, Behrooz MA. A Monte Carlo Study on Dose Enhancement by Homogeneous and Inhomogeneous Distributions of Gold Nanoparticles in Radiotherapy with Low Energy X-rays. J Biomed Phys Eng. 2018;8:13-28. PubMed PMID: 29732337; PubMed Central PMCID: PMC5928303.

13. Rezaei H, Zabihzadeh M, Ghorbani M, Goli Ahmadabad F, Mostaghimi $\mathrm{H}$. Evaluation of dose enhancement in presence of gold nanoparticles in eye brachytherapy by (103)Pd source. Australas Phys Eng Sci Med. 2017;40:545-53. doi: 10.1007/s13246-017-0555-1. PubMed PMID: 28509080.

14.Abdelhalim MA, Mady MM, Ghannam MM. Rheological and dielectric properties of different gold nanoparticle sizes. Lipids Health Dis. 2011;10:208. doi: 10.1186/1476-511X10-208. PubMed PMID: 22078458; PubMed Central PMCID: PMC3231822.

15.Callaghan MF, Lund T, Hashemzadeh P, Roitt IM, Bayford RH, editors. An investigation of the impedance properties of gold nanoparticles. Journal of Physics: Conference Series; 2010;224:012058.

16.Khalafalla $M$, Mesli $A$, Widattallah $H$, Sellai A, Al-Harthi S, Al-Lawati HA, et al. Sizedependent conductivity dispersion of gold nanoparticle colloids in a microchip: contactless measurements. Journal of nanoparticle research. 2014;16:2546.

17. Griffiths $H$. A phantom for electrical impedance tomography. Clin Phys Physiol Meas. 1988;9 Suppl A:15-20. PubMed PMID: 3240643.

18. Lee KH, Kim YT, Oh TI, Woo EJ, editors. Complex conductivity spectra of seven materials and phantom design for EIT. 13th International Conference on Electrical Bioimpedance and the 8th Conference on Electrical Impedance Tomography; 2007: Springer.

19.Bera TK, Nagaraju J. A chicken tissue phantom for studying an electrical impedance tomography (EIT) system suitable for clinical imaging. Sensing and Imaging: An International Journal. 2011;12:95-116.

20.Bera TK, Nagaraju J. Electrical impedance spectroscopic studies on broiler chicken tissue suitable for the development of practical phantoms in multifrequency EIT. Journal of Electrical Bioimpedance. 2011;2:48-63.

21.Bera TK, Nagaraju J. Studying the resistivity imaging of chicken tissue phantoms with different current patterns in Electrical Impedance Tomography (EIT). Measurement. 2012;45:663-82.

22. Dean D, Machado-Aranda D, Ramanathan T, Molina I, Sundararajan R, editors. Electrical properties of biological tissues-an impedance spectroscopy study. Electrical Insulation and Dielectric Phenomena, 2006 IEEE Conference on; 2006: IEEE.

23.Okazaki K, Tangoku A, Morimoto T, Kotani R, Hattori K, Yasuno E, et al. Basic study of a diagnostic modality employing a new electrical impedance tomography (EIT) method for noninvasive measurement in localized tissues. $J$ Med Invest. 2010;57:205-18. PubMed PMID: 20847519.

24.Bera TK. Bioelectrical Impedance Methods for Noninvasive Health Monitoring: A Review. J Med Eng. 2014;2014:381251. doi: $\quad 10.1155 / 2014 / 381251$. PubMed PMID: 27006932; PubMed Central PMCID: PMC4782691.

25.Kimling J, Maier M, Okenve B, Kotaidis V, Ballot $H$, Plech A. Turkevich method for gold nanoparticle synthesis revisited. J Phys Chem B. 2006;110:15700-7. doi: 10.1021/ jp061667w. PubMed PMID: 16898714.

26. Ali H, Karim S, Rafiq M, Maaz K, ur Rahman A, Nisar A, et al. Electrical conduction mecha- 
nism in ZnS nanoparticles. Journal of alloys and compounds. 2014;612:64-8.

27.Pfeiffer C, Rehbock C, Huhn D, Carrillo-Carrion $\mathrm{C}$, de Aberasturi DJ, Merk V, et al. Interaction of colloidal nanoparticles with their local environment: the (ionic) nanoenvironment around nanoparticles is different from bulk and determines the physico-chemical properties of the nanoparticles. $J R$ Soc $I n-$ terface. 2014;11:20130931. doi: 10.1098/ rsif.2013.0931. PubMed PMID: 24759541; PubMed Central PMCID: PMC4032524.

28.Dean DA, Ramanathan T, Machado D, Sundararajan R. Electrical Impedance Spectroscopy Study of Biological Tissues. J Electrostat. 2008;66:165-77. doi: 10.1016/j. elstat.2007.11.005. PubMed PMID: 19255614; PubMed Central PMCID: PMC2597841. 